\title{
Descripción y propuesta de manejo agroecológico de la subcuenca del río Babahoyo, Ecuador
}

\section{Description and agroecological management proposal of the Babahoyo River sub-basin, Ecuador}

\author{
Oscar Caicedo-Camposano $^{1 *}$, Darío Dueñas-Alvarado ${ }^{1}$, John Franco-Rodríguez $^{2}$, Ángel Triana-Tomalá $^{2}$ \\ ${ }^{1}$ Universidad Técnica de Babahoyo \\ ${ }^{2}$ Universidad Católica de Santiago de Guayaquil \\ *ocamposano@utb.edu.ec
}

DOI: https://doi.org/10.26871/killkana_tecnica.v3i3.577

\begin{abstract}
Resumen
La subcuenca del río Babahoyo es parte de la hidrografía de la cuenca del río Guayas puesto que el río Babahoyo junto con el río Daule son los dos afluentes que forman el gran río Guayas. Esta subcuenca recorre la zona este de la provincia de Los Ríos, comprende una superficie de 2.940,18 km2 que representa el 41,90\% del total de la subcuenca del río Babahoyo. Las características climáticas de la subcuenca del río Babahoyo son $25,5^{\circ} \mathrm{C}$ de temperatura media anual, con un $83 \%$ de humedad relativa, 1.050 horas de brillo solar, $1,3 \mathrm{~m} / \mathrm{s}$ de velocidad del viento y una precipitación de 2.200 milímetros. La agricultura es la principal actividad económica y fuente de producción de la subcuenca del río Babahoyo. Los suelos que tienen son de usos simples o solos, y ocupan el $37 \%$ de la superficie, mientras que las coberturas y usos asociados corresponden al $47 \%$; y otros tipos de cobertura ocupan el $16 \%$ como son: asentamientos poblados, bosques naturales e intervenidos, cultivos transitorios, cuerpos de agua, entre otros. La propuesta contempla entre otras cosas, promover una intensiva educación ambiental, un manejo adecuado de la ganadería, la conservación de la calidad de las aguas y la diversidad biológica y genética, así como reemplazar la agricultura convencional por una agricultura sustentable que incluya prácticas agroecológicas en los sistemas de cultivos. El objetivo de este trabajo fue el de caracterizar y proponer un manejo agroecológico de la subcuenca del río Babahoyo.
\end{abstract}

Palabras clave: Caracterizar, subcuenca, manejo agroecológico.

\begin{abstract}
The Babahoyo River sub-basin is part of the Guayas River basin hydrography, as the Babahoyo River, and the Daule River, are the two tributaries forming the great Guayas River. This sub-basin covers the eastern part of Los Ríos province, in an area of 2,940.18 km2, representing 41.90\% of the total area of the Babahoyo River sub-basin. The weather in the Babahoyo River sub-basin is characterized by an annual average temperature of $25.5^{\circ} \mathrm{C}$, with $83 \%$ of relative humidity, 1,050 hours of sunshine, $1.3 \mathrm{~m} / \mathrm{s}$ wind speed, and 2,200 millimetres of rain precipitation. Agriculture is the main economic activity and production source of the Babahoyo River sub-basin. Single-use soils occupy $37 \%$ of the surface, while coverings and associated uses correspond to 47\%; other types of coverings such as populated settlements, natural and intervened forests, transitory crops, bodies of water, among others occupy $16 \%$ of the area. The proposal considers, among other things, the promotion of intensive environmental education, proper livestock management, water quality, biological, and genetic diversity conservation; as well as the replacement of conventional agriculture by sustainable agriculture that involves agroecological practices in crop systems. This study aimed to characterize and propose the agroecological management of the Babahoyo River sub-basin.
\end{abstract}

Keywords: Characterize, sub-basin, agroecological management.

\section{INTRODUCCIÓN}

La subcuenca del río Babahoyo es parte de la hidrografía de la cuenca del río Guayas puesto que el río Babahoyo junto con el río Daule son los dos afluentes que forman el gran río Guayas; es decir que la subcuenca del Babahoyo se ubica entre las provincias de Los Ríos, Guayas, Cotopaxi,
Bolívar, Manabí, Cañar, Chimborazo y Santo Domingo, en el centro occidental del Ecuador.

Limita al norte con la cuenca del río Esmeraldas; al sur con las cuencas de los ríos Zapotal, Taura, Cañar y Santiago; al este con las cuencas de los ríos Esmeraldas y Pastaza; y al oeste con las cuencas del Jama, Chone, Portoviejo y Jipijapa. Se extienden entre los paralelos $00^{\circ} 14^{\prime} \mathrm{S}, 02^{\circ} 27^{\prime} \mathrm{S}$ 
y los meridianos $78^{\circ} 36^{\prime} \mathrm{W}, 80^{\circ} 36^{\prime} \mathrm{W}$ como se muestra en la Figura 1.

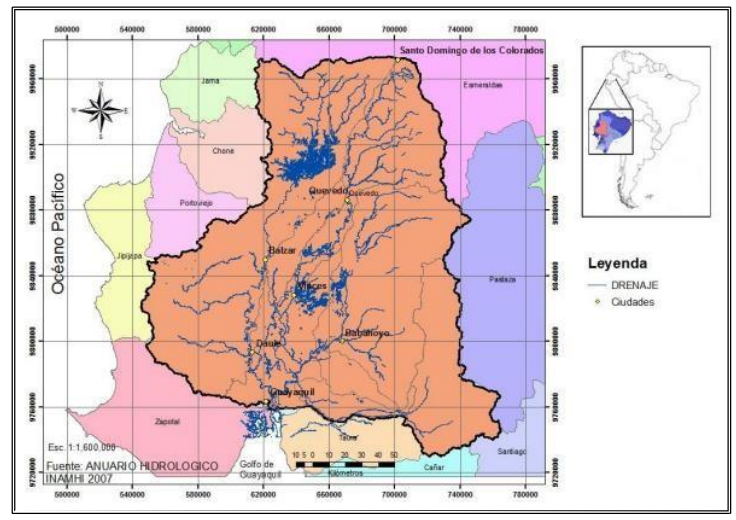

Fig. 1: Ubicación de la cuenca del Río Guayas

Esta cuenca hidrográfica caracteriza por la gran variedad e intensa actividad agrícola, ganadera, forestal, acuícola, pesquera, etcétera; todo esto gracias a la excelente calidad de sus suelos y a la dinámica de la tierra con el mar en la zona estuarina, que la ubica como el mayor centro de producción de bienes agropecuarios a nivel nacional, tanto para el mercado interno como para el externo a través de las exportaciones desde los puertos marítimos del Ecuador.

La cuenca del rio Guayas pertenece a la vertiente del Pacífico, con un área aproximada de $34.500 \mathrm{~km} 2$, favorece a la generación de energía hidroeléctrica para el Ecuador. La superficie de la cuenca corresponde al $12,57 \%$ del territorio patrio, la población que habita en este territorio se estima en 5'592.025 habitantes, representado al 39,37\% del total de la población ecuatoriana.

\section{CARACTERIZACIÓN DE LA SUBCUENCA DE RÍO BABAHOYO}

Esta subcuenca recorre la zona Este de la provincia de Los Ríos, comprende una superficie de 2.940,18 km2 que significa el 41,90\% del total de la subcuenca del río Babahoyo y la integran las microcuencas: río Chipe, río Lechugal, estero Calabicito, río Oncebí, río Jordán, rio de las Piedras, río Pijullo, río el Playón, río el Tilimbala, río La Esmeraldas, Estero de Damas, río El Tigrillo, río Las Juntas, río San Antonio, Río Viejo, río Cristal y drenajes menores.

\section{A. Clima de la subcuenca del Babahoyo}

Los promedios anuales de las variables climáticas de la subcuenca del rio Babahoyo son 25,5 $\mathrm{C}$ de temperatura media anual, $83 \%$ e humedad relativa, 1.050 horas de brillo solar, $1,3 \mathrm{~m} / \mathrm{s}$ de velocidad del viento y una precipitación de 2.200 milímetros.

El clima en gran parte del territorio se comporta de manera homogénea, de conformidad con las regiones climáticas del Ecuador propuestas por Pourrut la mayor parte del territorio de la cuenca presenta un clima Tropical Megatérmico Semi - Húmedo, que se caracteriza por registrar únicamente una estación húmeda y una estación seca, muy marcadas con un numero de meses húmedos y secos en el año, estos son de enero a abril y de mayo a diciembre correspondientemente, acompañada de temperaturas medias superiores a $22^{\circ} \mathrm{C}$ lluvias que van desde $500 \mathrm{~mm}$ a $2000 \mathrm{~mm}$ de promedio anual.

Luego tenemos un clima Tropical Megatérmico Húmedo teniendo influencia directa en el cantón Valencia y las partes altas de los cantones Montalvo y Urdaneta, dicha zona se caracteriza por tener una estación seca que van de seis a ocho meses secos en el año y una estación lluviosa con precipitaciones entre $1000 \mathrm{~mm}$ a $2000 \mathrm{~mm}$, con temperaturas medias superiores a $22^{\circ} \mathrm{C}$.

La estación húmeda está comprendida entre los meses de enero a abril, mientras que la estación seca se presenta desde mayo hasta diciembre.

\section{B. Recursos hídricos de la subcuenca del Babahoyo}

La subcuenca del río Babahoyo tiene una superficie de $7.827 \mathrm{~km} 2$ y constituye casi un cuarto de la superficie total de la cuenca del Guayas; está localizada en el sector centraleste de dicha cuenca hidrográfica y básicamente la integran dos ríos que se originan en la cordillera de Los Andes: el río Catarama que nace en e 1 extremo Noreste de la subcuenca y tiene una dirección de desarrollo francamente de NorteSur, y el río San Pablo de Las Juntas, que nace en e 1 sureste y tiene un pronunciado desarrollo Este - Oeste hasta que se une con el río Catarama para juntos iniciar el río Babahoyo. Los resultados de la investigación de CaicedoCamposano et al. (2019)[1] indican que en la época seca las aguas de la subcuenca del rio Babahoyo no son aptas para la agricultura, no obstante, en este período se deben garantizar las necesidades hídricas de los cultivos con el riego.

\section{Uso del suelo en la subcuenca del Babahoyo}

En la subcuenca del río Babahoyo, los suelos de usos simples o solos, ocupan el $37 \%$ de la superficie, mientras que las coberturas y usos asociados corresponden al $47 \%$ y otros tipos de cobertura ocupan el $16 \%$ como son; asentamientos poblados, bosques naturales e intervenidos, cuerpos de agua, ciclo corto, pastos naturales y vegetación arbustiva.

TABLA I: Áreas de coberturas según el uso del suelo (GAD Provincial de Los Ríos, 2016)

\begin{tabular}{lcc}
\hline Uso Suelo & Superficie (ha) & Porcentaje \% \\
\hline Arboricultura & $11.325,8$ & 1,57 \\
Arboricultura - Pastos plantados & $50.845,5$ & 7,06 \\
Arroz & $125.322,2$ & 17,39 \\
Arroz - Pastos plantados & $75.241,5$ & 10,44 \\
Asentamiento poblado & $2.183,9$ & 0,30 \\
Banano & $26.527,9$ & 3,68 \\
Bosque natural & $44.092,50$ & 6,12 \\
Bosque natural - Arboricultura & 0 & 0 \\
\hline
\end{tabular}


TABLA II: Áreas de coberturas según el uso del suelo (GAD Provincial de Los Ríos, 2016)...(continuación)

\begin{tabular}{lcc}
\hline Uso Suelo & Superficie (ha) & Porcentaje \% \\
\hline Bosque natural - Pastos plantados & $1.788,20$ & 0,25 \\
Bosque natural intervenido & $15.864,80$ & 2,2 \\
Cacao & $17.431,60$ & 2,42 \\
Café & $7.698,10$ & 1,07 \\
Café - Cacao & $71.384,90$ & 9,91 \\
Caña de azúcar & $1.981,00$ & 0,27 \\
Cuerpos de agua & $12.765,20$ & 1,77 \\
Cultivos de ciclo corto & $38.445,50$ & 5,34 \\
Cultivos de ciclo corto - Arboricultura & $16.545,30$ & 2,3 \\
Cultivos de ciclo corto - Pastos plantados & $114.817,80$ & 15,94 \\
Desconocido & 26,3 & 0 \\
Frutales - Pastos plantados & $4.610,00$ & 0,64 \\
Maíz & $6.906,90$ & 0,96 \\
Palma africana & $26.553,00$ & 3,69 \\
Pastos naturales & 945,2 & 0,13 \\
\hline
\end{tabular}

\section{Población de la subcuenca del Babahoyo}

Según los estudios realizados por la Comisión de Estudios para el Desarrollo de la Cuenca del río Guayas (CEDEGE) en conjunto con la CEPAL, para el año de 1983 la población en la subcuenca del río Babahoyo era de 322.279 habitantes, en la actualidad según los datos recabados en el portal del Instituto Nacional de Estadísticas y Censos esa cifra se ha incrementado en algo más del $200 \%$, es decir que los habitantes en este territorio son algo más de 700.000, más del $40 \%$ reside en el área rural que es donde se genera la economía, puesto que por su ubicación geográfica, la características del suelo y las condiciones climáticas, la agricultura es el motor económico de la subcuenca del río Babahoyo [2].

En el gobierno del Econ. Rafael Correa Delgado, todos los habitantes de escasos recursos económicos de este territorio y del resto del país tuvieron acceso a viviendas de hormigón armado, por lo que es poco común observar viviendas de madera o caña en los campos del Ecuador.

En lo referente a las escuelas y colegios, en eso si el gobierno del citado economista construyó las denominadas "Escuelas del Milenio" y los "Colegios Replicas" en los principales centros poblados de casi todas las provincias del Ecuador.

En el caso de la subcuenca del Babahoyo, sus habitantes cuentan con la Universidad Técnica de Babahoyo y Universidad Regional Autónoma de Los Andes.

\section{E. Biodiversidad de la subcuenca del Babahoyo}

La vegetación natural es la selva, de la cual se explotan las especies madereras como caoba, cedro y laurel. En varias zonas crecen los lechuguines de flores de color violeta y otras plantas acuáticas. Existe también una variedad de especies animales como: monos, aves de corral, zorros, iguanas, cusumbo, guanta, guatusa, chorongo llamado Pancho, ovejas, chivos cruzados; aves acuáticas como el pato cuervo, papagayos, ardillas, comadrejas, murciélagos, tigrillos, gavilanes entre otros [3].
Estas especies y otras, las encontramos en todo el territorio de la subcuenca del río Babahoyo. Actualmente algunas especies de flora y fauna se han extinguido y otras están en peligro de extinción; esto a consecuencia de por la forestación indiscriminada y la caza ilegal y la expansión de la frontera agrícola [3].

\section{F. Actividades económicas en la subcuenca del Babahoyo}

La agricultura es la principal actividad económica y fuente de producción de la subcuenca del Babahoyo. En las llanuras existen cultivos de arroz, de caña de azúcar, maíz, palma africana, maracuyá, papaya, etc. y en las partes más altas hay condiciones excelentes para los cultivos de exportación: café, cacao, banano, plátano, entre otros. La actividad desplegada por la Universidad Técnica de Babahoyo ha permitido un mejoramiento de la productividad agrícola gracias al empleo de la técnica, fertilizantes, maquinaria y semillas seleccionadas. La pesca también es una actividad de la que subsisten algunos pobladores en diferentes localidades ya que gracias a la extensa red fluvial de la subcuenca abundan diferentes variedades de peces, entre los que se puede citar: róbalos, sábalos, bocachicos, bagres, lisas. Estos se pescan durante todo el año y se destinan para consumo humano. Las labores de pesca se realizan a nivel artesanal mediante la utilización de pequeñas embarcaciones y el manejo de anzuelos, redes, atarrayas y bajíos.

Una actividad recientemente explotada es el turismo, los campos cultivados, las haciendas, los ríos, las costumbres montubias, son atractivos indudables para el visitante. Existen además sitios ideales para la caza y la pesca. Los principales centros de atracción turística son: Para disfrutar del río, las playas de la hacienda El Salto y las del Río Seco y El Cerro Cacharí [4].

Otras fuentes de empleo son las instituciones de Estado tales como Gobernación de Los Ríos, Gobierno Autónomo Descentralizado Provincial de Los Ríos, Municipalidad de Babahoyo, Instituciones de Educación Básica, Instituciones de Educación Media, Instituciones de Educación Superior, y oficinas de Direcciones Provinciales de algunos Ministerios [5].

\section{PRINCIPALES PROYECTOS DESARROLLADOS EN LA SUBCUENCA DEL BABAHOYO}

\section{A. Sistema de Riego y Drenaje Babahoyo}

El Gobierno Nacional con el financiamiento del Banco Interamericano de Desarrollo (BID), el aval técnico de la Comisión de Estudios para el Desarrollo de la Cuenca del río Guayas (CEDEGE) y la contratación de la compañía constructora Hidalgo - Hidalgo, implantó entre los años 1975 a 1979, en la provincia de Los Ríos el Sistema de riego y drenaje Babahoyo, que está ubicado entre las coordenadas geográficas $1^{\circ} 48^{\prime}$ hasta $1^{\circ} 55^{\prime}$ latitud sur y $79^{\circ} 30^{\prime}$ hasta $79^{\circ} 18^{\prime}$ longitud oeste.

El citado Sistema fue construido para el desarrollo agrícola bajo riego (con control de inundaciones) de 9000 has. 
netas, con un patrón de cultivos representado básicamente por el arroz, para lo cual se implementaron las siguientes obras físicas:

- Dos estructuras derivadoras con sus respectivas tomas; la No. 1 ubicada en el río Grande de las Juntas, para dotar de riego a 4000 has. netas (Área A) y la No. 2 ubicada en el río San Pablo para servir a 5000 has. netas (Área B).

- Un sistema de conducción de agua por gravedad con un total de $111 \mathrm{~km}$ de canales principales y secundarios revestidos con un recubrimiento de hormigón simple de 7 y $5 \mathrm{~cm}$, respectivamente, de los cuales $45 \mathrm{Km}$ corresponden al área A y $66 \mathrm{Km}$ al área B.

- Una red de canales de drenaje primarios y secundarios con una longitud de $102.8 \mathrm{Km}$, de los cuales 54.2 $\mathrm{Km}$ corresponden al área $\mathrm{A}$ y $48.6 \mathrm{Km}$ al área $\mathrm{B}$, que desembocan en un colector de $5.3 \mathrm{Km}$.

- Una red de $125 \mathrm{Km}$ de caminos parcelarios, de los cuales $36 \mathrm{Km}$ están estabilizados con doble sello asfáltico y $89 \mathrm{Km}$ son lastrados.

- Una estación de bombeo para descargar hacia el río San Pablo con un caudal de $18.75 \mathrm{~m} / \mathrm{s}$ mediante 5 unidades, el agua que, durante la etapa de lluvias, se acumula dentro del Sistema de riego.

- Diques marginales en todo el perímetro del Sistema, en una longitud de $63.16 \mathrm{Km}$ para el control de las inundaciones.

- Sistematización parcelaria de 4500 has.

La operación y mantenimiento del Sistema de riego fue realizado por CEDEGE hasta el 26 de noviembre de 1999, fecha en la cual el Gobierno Nacional, por intermedio del Ministerio de Agricultura, realizó la transferencia de las actividades antes mencionadas a los usuarios (922 según padrón) agrupados con una Junta General, a quien se entregó en comodato edificios, maquinaria y equipos que habían cumplido su vida útil.

Este proyecto jamás se ejecutó al $100 \%$, puesto que, durante la construcción del mismo, se incorporaron 2000 hectáreas (Área C) a los diseños iniciales, lo que daría un total de 11.000 hectáreas de riego. Las obras hidráulicas de captación, conducción y distribución de agua se ejecutaron únicamente para las áreas A y B, sin embargo, jamás las para riego a nivel de finca, es decir, la sistematización parcelaria se hizo apenas para algo más de 5000 hectáreas, razón por la cual algunos usuarios costearon los trabajos de sistematización parcelaria para sus predios agrícolas.

Con todo ese antecedente la zona del Sistema de Riego y Drenaje Babahoyo es el lugar de mayor producción arrocera durante la estación seca en subcuenca del Babahoyo. Actualmente la Junta General de Usuarios es quien administra el mencionado distrito de riego con el apoyo y acompañamiento del GAD de Los Ríos [6].

\section{B. Sistema de Riego y Drenaje Catarama}

El Sistema de riego y Drenaje Catarama irrigaría 5.760 hectáreas, esta obra de infraestructura agrícola tuvo como objetivos el mejoramiento y tecnificación de la productividad; incremento de la producción de los sustitutos de importación; activación de la economía en el área del proyecto; y la ampliación de las metas de los agricultores en las labores agrícolas.

El sistema de riego y drenaje consta de los siguientes elementos:

- $\quad$ Sobre el río Sibimbe una presa derivadora de 47 metros de longitud y 4.20 metros de altura. Tiene dos compuertas de limpieza, una estructura equipada con dos compuertas deslizantes, un sedimentador con desagüe de limpieza por gravedad.

Un dispositivo de medición de caudal, un paso de peces para el mantenimiento del río y trabajos, más una estructura que suministrará 4.85 metros cúbicos por segundo, para el riego de 3,730 de hectárea.

- Una estación de bombeo está localizada sobre el margen derecho del río Catarama, la cual consta de tres bombas de flujo mixto y de eje vertical, con una carga total de 14.2 metros. Tiene tres motores eléctricos de 250 kilovatios por unidad, instalaciones eléctricas, un transformador principal de mil kilovatios con una capacidad de bombeo de 2,7 metros cúbicos por segundo que permitirá el riego de 2,030 hectáreas.

- $\quad$ Sistema de riego.

Canales de riego completamente revestido de hormigón, los mismos que tienen una longitud total de 67, 1 $\mathrm{km}$.

Las estructuras afines del sistema de riego de todo el proyecto suman 314 unidades, en que incluyen 91 tomas, 46 estructuras de control, 67 alcantarillas, un sifón invertido, 41 caídas, 11 aliviaderos, 43 puentes y otras 14 estructuras.

- $\quad$ Sistema de Drenaje.

El sistema de drenaje tiene $69 \mathrm{~km}$. de longitud de canales, en este sistema todos los canales fueron construidos en tierra con sección trapezoide, existen 262 estructuras afines clasificadas en 75 alcantarilladas, 43 caídas, 112 entradas de drenaje, 7 cruces de drenaje, 21 puentes, 2 compuertas de drenaje y otras 2 estructuras.

- $\quad$ Red de caminos.

Los caminos principales y secundarios suman 137 kilómetros y corren a lo largo de los canales de riego y drenaje, todas las superficies de los caminos son pavimentada.

- Los caminos principales son asfaltados en una longitud de 18,5 kilómetros, y los secundarios quedaron a nivel de sub base.

En el año 2003 se entregó la obra terminada de este sistema de riego, aunque la realidad es que jamás se ejecutaron por completo las obras a nivel de finca, razón por la cual hasta el año 2013 únicamente se estuvieron irrigando 2.081 hectáreas, ya que el préstamo para el proyecto financio solamente el diseño y construcción de la red de distribución para el riego únicamente, pues se consideró que las 3.679 hectáreas (obras a nivel de finca) restantes debían 
ser construidas por los propios agricultores. A pesar de las múltiples labores que el GAD de Los Ríos realiza en este distrito de riego, este sistema hasta la actualidad no opera a toda su capacidad, pues aún faltan algunas obras a nivel de predios agrícolas, lo cual par algunos productores agrícolas es un severo problema por la falta de infraestructura en sus propiedades [7]

\section{Proyectos desarrollados por la Cámara de la industria de la Ciencia de los Cultivos "InnovAgro Ecuador" \\ 1. Programa CuidAgro}

El Programa CuidAgro promueve el uso responsable de productos fitosanitarios en el territorio de la subcuenca del río Babahoyo ofreciendo cursos de capacitación y entrenamiento para instructores, agricultores, expendedores, exportadores, amas de casa, estudiantes, agrónomos, médicos y personal de enfermería.

CuidAgro se desarrolla con múltiples alianzas y cuenta con la activa participación de la Agencia Ecuatoriana de Aseguramiento de la Calidad del Agro, AGROCALIDAD, Los Ministerios de Salud, del Ambiente, de Agricultura, Universidades, Organizaciones no Gubernamentales, Alcaldías o Municipalidades, Gremios Agrícolas y Agencias de Cooperación internacional.

Entre el 2012 y 2016 el programa CuidAgro logró capacitar y entrenar a más de 45.000 personas en el uso correcto de productos agrotóxicos.

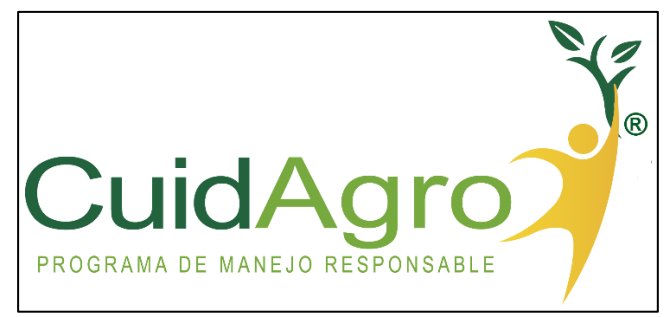

Fig. 2: Programa CuidAgro que se ejecuta en el Proyecto de Riego Babahoyo

\section{Programa Campo Limpio}

CampoLimpio es el Programa de Manejo de Envases Vacíos, que promueve la devolución de los envases con la técnica del Triple Lavado y perforados para evitar su reutilización y poder proceder con una adecuada disposición final.

CampoLimpio cuenta con Centros de Acopio Primarios y Centros de Acopio Temporales, equipados con maquinaria especial para el acondicionamiento de los envases recuperados, como compactadoras o trituradoras.

Durante el año 2015 se logró recuperar 123,9 toneladas de envases vacíos gracias a la participación de algunas de las empresas miembros que recuperaron de manera directa 54 toneladas, logrando recuperar el mínimo establecido por el Ministerio del Ambiente, y un $18 \%$ adicional en relación con el año anterior.
Cabe mencionar que la Junta General de Usuarios del Sistema de Riego y Drenaje Babahoyo cedió un lote de terreno para montar un centro de acopio de envases vacíos de agrotóxicos como contraparte de la alianza con InnovAgro y la Prefectura.

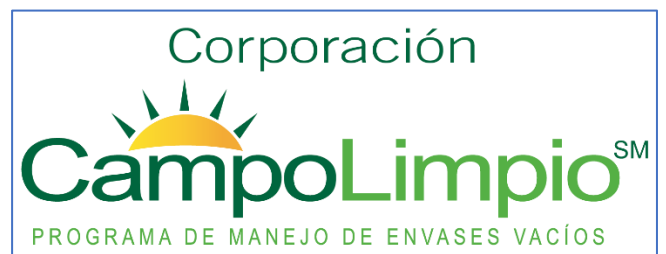

Fig. 3: Programa Campo Limpio que se ejecuta en el Proyecto de Riego Babahoyo

D. Proyectos agropecuarios estatales de los cuales se benefician los habitantes en el territorio de la subcuenca del rio Babahoyo

1. Proyecto Nacional de Innovación Tecnológica Participativa y Productividad Agrícola (PITPPA)

Tiene objetivo promover la reactivación del agro, a través de la optimización de procesos de asistencia técnica y extensionismo, complementando con dotación de tecnología innovadora, infraestructura y equipamiento tecnológico de punta a fin de mejorar las capacidades productivas tradicionales de los pequeños y medianos productores del sector agropecuario, propendiendo que la población beneficiaria mejore su calidad de vida.

El proyecto está orientado a elevar la productividad nacional en los rubros banano, arroz, plátano, papa, maíz duro, trigo, cebada, soya, maíz suave choclo, frutales, café, cacao, hortalizas entre otras, en predios de pequeños y medianos productores, así como a recuperar los sistemas integrales o encadenamientos internos; es decir, la relación entre la producción agrícola y la pecuaria en el manejo y reciclaje de desechos.

Se ejecuta a las 24 provincias del Ecuador. Los beneficiarios son pequeños productores; es decir, los que poseen de 0 a 10 hectáreas.

Además, busca desarrollar procesos de innovación tecnológica que permitan mejorar los rendimientos agros productivos; implementar un sistema de asistencia técnica y extensión rural participativa, que desarrolle las capacidades agroproductivas y el tejido social de los pequeños y medianos productores.

También, dotar de infraestructura y equipamiento productivo para el mejoramiento de la competitividad sistémica de los productores; así como fortalecer la producción rural y la agricultura familiar campesina, de pequeños y medianos productores, mediante la innovación tecnológica que contribuya a la inclusión de los campesinos al sistema productivo, promoviendo el uso apropiado del suelo y prácticas agronómicas eficientes. 


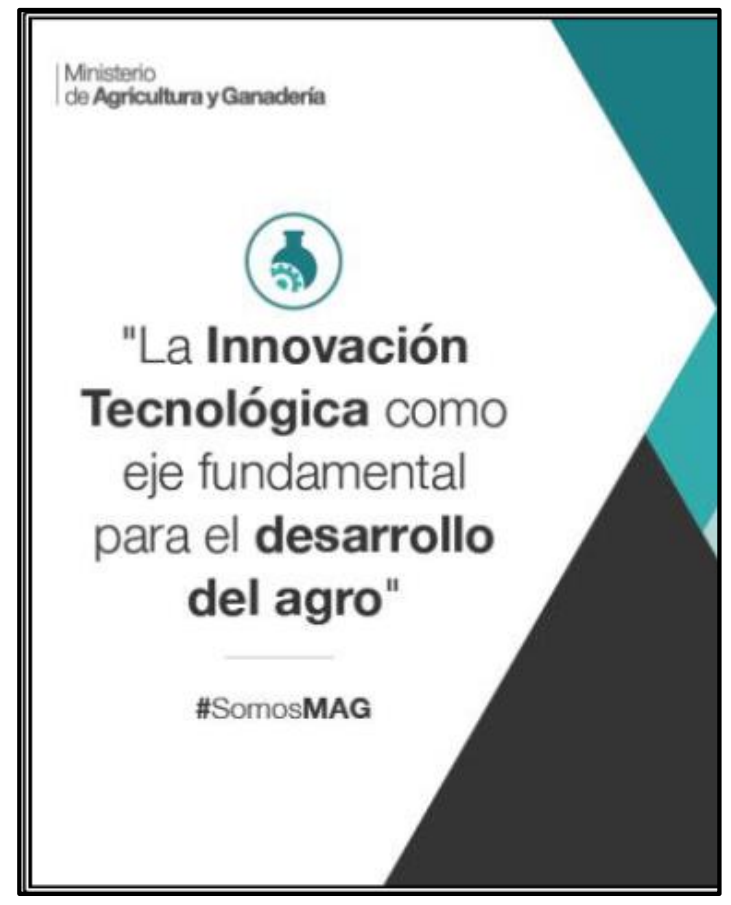

Fig. 4: Publicidad del PITPPA en el website del MAG del Ecuador

Este proyecto a pesar de llevar un corto tiempo en ejecución, hace pocos meses el gobierno nacional incrementó su presupuesto, no obstante, los resultados han sido negativos puesto que su ejecución se volvió más lenta después de haber obtenido mayor presupuesto, aun no se logra apreciar algún impacto en la población rural de la subcuenca del río Babahoyo [8].

\section{Proyecto AGROSEGURO para pequeños y medianos} productores y pescadores artesanales del Ecuador

Este proyecto tiene como objetivo implementar un sistema permanente de seguro subvencionado por el Estado, en beneficio de pequeños y medianos productores agropecuarios y pescadores artesanales del Ecuador, que les permita contratar pólizas de protección contra pérdidas de sus producciones, ocasionadas fundamentalmente por eventos climáticos y biológicos.

El Gobierno Ecuatoriano subsidia el $60 \%$ del valor de la prima neta del seguro. Los cultivos cubiertos por el seguro son los siguientes: arroz, banano, caña de azúcar, fréjol, maíz duro, maíz suave, papa, soya, tomate de árbol y trigo. El seguro cubre por diferentes eventos climatológicos y biológicos como son: sequia, inundación, exceso de humedad, vientos fuertes, incendio, helada, granizada, plagas incontrolables, enfermedades incontrolables, deslizamiento y taponamiento. Para el año 2016, el seguro agrícola ampliará su cobertura a los siguientes cultivos: café, cacao, haba, quinua, plátano y cebada.

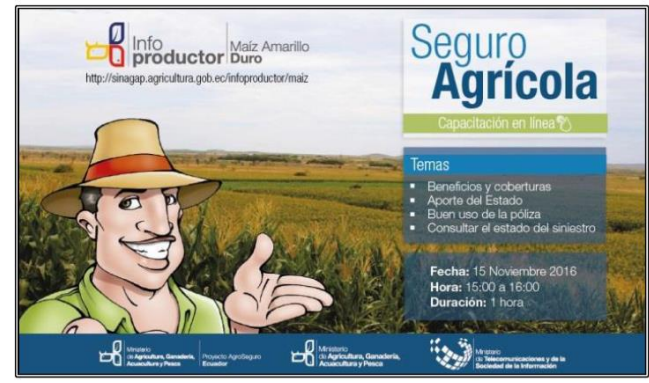

Fig. 5: Publicidad del PITPPA en el website del MAG del Ecuador

El Estado Ecuatoriano motivado en las perdidas agropecuarias que provoca el Fenómeno El Niño y los años anómalos (exageradamente húmedos), tuco la iniciativa de implementar este proyecto, el cual inicialmente no tuvo mucha aceptación por parte de los pequeños productores de gramíneas, sin embargo, hoy tanto los técnicos del Ministerio de Agricultura y productores trabajan de manera organizada para asegurar cultivos de ciclo corto y en casos muy raros aseguran plantaciones bananeras. El bananero no se ve muy beneficiado de este seguro ya que en el territorio de esta subcuenca predominan grandes bananeros con superficies superiores a 100 has [8].

\section{Proyecto: Implementación de nuevos silos de almace- namiento}

Tiene el propósito de aumentar la capacidad de almacenamiento público a través de la construcción de nuevas plantas de silos o de la ampliación de las existentes, lo que permitirá el control de precios en zonas productoras con alta concentración de pequeños agricultores de arroz y maíz, de esta manera se implementará también se implementará un sistema integral de compras, estableciéndose así establecido un sistema económico social, solidario y sostenible.

La entidad encargada de estos procesos es la Empresa Publica Unidad nacional de Almacenamiento EP (UNA EP), esta es una empresa pública vinculada con el Ministerio de Agricultura y es la responsable del almacenamiento y comercialización arroz, maíz y soya.

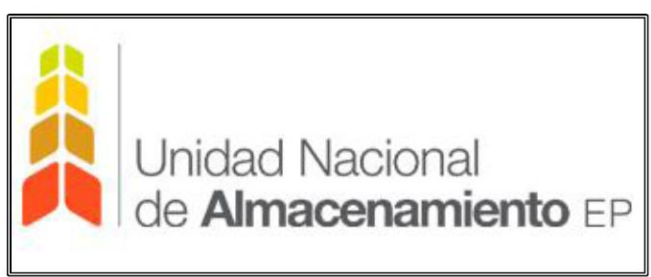

Fig. 6: Logo de la Unidad Nacional de Almacenamiento EP

Desde la creación de la UNA EP a pesar de los esfuerzos y la voluntad política del ministro de turno, esta entidad no ha logrado acopiar las cosechas de todos los productores 
de gramíneas, y aunque el gobierno establezca precios oficiales para las cosechas de arroz, maíz y soya, la UNA EP no brinda una atención integral, en donde no se vea perjudicado el productor; más bien sucede que los primeros agricultores que cosechan se ven beneficios con el precio oficial de determinado producto agrícola, y a medida que pasan los días, quienes llegan a vender sus cosechas se ven afectados en cuanto a precio, pues a estos ya no se les paga el precio oficial, sino que reciben valores por debajo de lo que el gobierno ha establecido, y luego hay quienes ni siquiera tienen la suerte de que su producto sea recibido.

\section{Proyecto de Reactivación de Café y Cacao Nacional fino de Aroma fue presentado a productores}

Este proyecto tiene como propósito impulsar por un período de diez años el Programa de Reactivación Cafetalera del Ecuador y el Programa de Reactivación del Sector Cacaotero Ecuatoriano, a través de incentivos productivos para la rehabilitación o establecimiento de cafetales tipo Arábigo y Robusta, y la renovación de plantaciones de Cacao Nacional Fino de Aroma.

El Ministerio de Agricultura, a través de la Subsecretaría de Agricultura, aplica sistemas productivos tecnificados y sostenibles. Brinda asistencia técnica, capacitación, incentivos a la productividad e investigación participativa directa con las organizaciones caficultoras y cacaoteras.

El fin es Lograr la participación al menos un $45 \%$ del mercado cafetero y cacaotero.

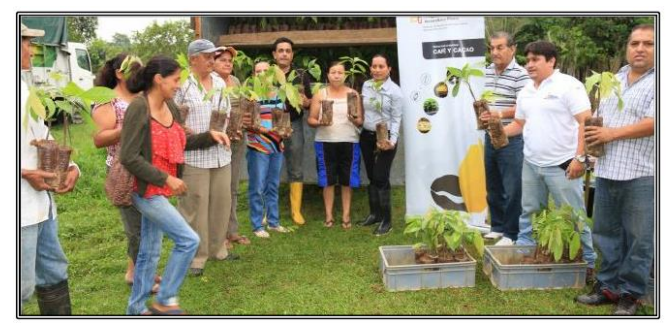

Fig. 7: Personal del MAG entregando plántulas en el marco de la Reactivación de Café y Cacao

\section{Plan de fomento de acceso a tierras de los productores} familiares del Ecuador (Plan Tierras)

Contribuir a reducir la inequidad de acceso a la tierra, para pequeños y medianos productores agropecuarios, mediante la adquisición, redistribución, legalización de predios estatales, privados y baldíos, utilizados por parte del estado, anclados planes, programas y proyectos productivos agropecuarios, que permitan el desarrollo productivo y bienestar de los productores y familias rurales.

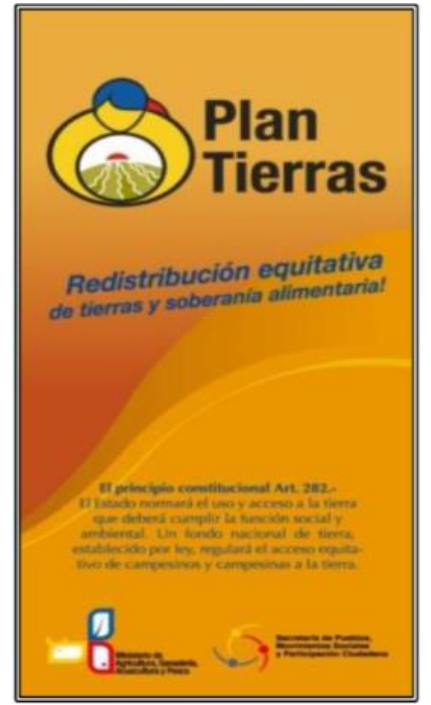

Fig. 8: Publicidad del Plan Tierras en el website del MAG del Ecuador

Aunque este proyecto tuvo gran expectativa, no se ha ejecutado conforme a lo planificado por falta de recursos financieros y personal.

\section{Proyecto Nacional de Semillas para agrocadenas es- tratégicas}

Incrementar la productividad en los cultivos de pequeños y medianos productores mediante la dotación de paquetes tecnológicos subsidiados que contienen semillas certificadas, fertilizantes, agroinsumos, asistencia técnica gratuita del MAGAP (ahora MAG) y seguro agrícola; garantizando disponibilidad, acceso y uso tecnificado de la semilla de alto rendimiento, aplicando soluciones integrales agrotécnicas en el manejo del cultivo y apoyando a la comercialización de la producción generada por los pequeños y medianos productores, logrando así la reducción de importaciones de productos primarios y potencializando el desarrollo del sector agrícola.

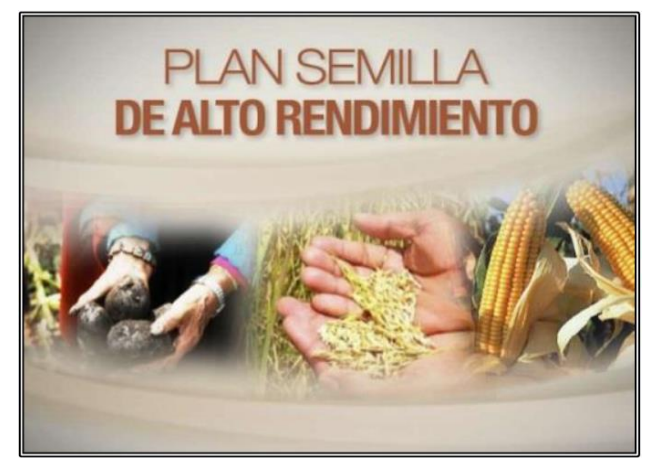

Fig. 9: Publicidad del Proyecto Nacional de Semillas para agrocadenas estratégicas 
El proyecto no tiene el dictamen favorable de la Secretaria Nacional de Planificación y Desarrollo.

7. Fomento a la producción agrícola a través del mejoramiento de la eficiencia de los sistemas de riego y drenaje a nivel nacional

Contribuir al incremento de la producción agrícola de pequeños productores rurales a través del uso y aprovechamiento agrícola y productivo del recurso hídrico y su participación en el seguimiento del Plan Nacional de Riego y Drenaje, que ejerce y ejecuta en calidad de ente rector de la política nacional agropecuaria.

Este proyecto arranco con buena expectativa, no obstante al momento en que el Gobierno Autónomo Descentralizado Provincial de Los Ríos (GAD Provincial o Prefectura) asumió la competencia de Riego y Drenaje indicada en el Artículo 263 inciso 5 de la Carta Magna del Ecuador, todos los trabajos de riego han estado a cargo de la Prefectura desde la Dirección de Riego, Drenaje y Dragas, aunque el trabajo que esta entidad realiza requiere de gran inversión, hasta el momento es notoria la labor de sus técnicos y sus maquinarias en la operación y mantenimiento de Sistemas de Riego y Drenaje Comunitarios [8].

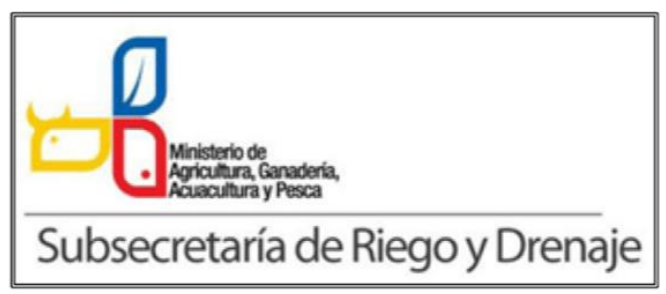

Fig. 10: Logo de la Subsecretaria de Riego y Drenaje del MAG

\section{Proyecto nacional de ganadería sostenible}

El Programa Ganadería Sostenible se enmarca en la actividad pecuaria de pequeños y medianos productores, con modelos de producción amigables con el ambiente. Promueve el desarrollo participativo e inclusivo para alcanzar los objetivos del Plan Nacional del Buen Vivir y la Soberanía Alimentaria. Es ejecutado por la Subsecretaría de Ganadería.

Contribuye a la seguridad e inocuidad alimentaria de la población ecuatoriana, a través del desarrollo y optimización de la productividad pecuaria del país, bajo la implementación de sistemas productivos tecnificados y eficientes de manera sustentable y sostenible, a fin de mejorar los ingresos de los pequeños y medianos productores del sector ganadero, incrementando su producción mediante la aplicación de mecanismos de identificación, trazabilidad, conservación de pastos, mejoramiento genético e implementación de centros de acopio que incrementen la producción pecuaria, fomentando la asociatividad y creación de cadenas cortas de comercialización.
A pesar de que la subcuenca del río Babahoyo se la explota al $100 \%$ en producción ganadera, el proyecto ha impacto positivo hasta ahora, sobre todo se ha visto un incremento en la actividad ganadera, la cual históricamente venía siendo poco atendida por el estado, el cual hace algunas décadas, únicamente tenía firmado un convenio con las asociaciones de ganaderos para que estos manejen la campaña para la erradicación de la fiebre aftosa.

\section{PRopuesta Para un MANEJO AgRoecológico DE LA SUBCUENCA DEL RÍO BABAHOYO}

La Constitución del Ecuador en su Art. 263 menciona que los GADs Provinciales tendrán como competencias, "Ejecutar, en coordinación con el gobierno regional, obras en cuencas y micro cuencas" y "La gestión ambiental provincial" por eso en uso de sus competencias constitucionales esta institución debería empezar abrir espacios multisectoriales de diálogo y concertación sobre el tema de cuencas hidrográficas para la elaboración de un Plan de Manejo Integrado de Cuencas con visión Agroecológica. En ellos deben participar los principales actores involucrados, de manera que se logre una mayor integración de planes, programas y proyectos, y sobre todo, de instrumentos de política a nivel de las cuencas hidrográficas.

A continuación, se exponen las propuestas para realizar un Manejo Agroecológico de la Subcuenca del Río Babahoyo:

- Instalar una red de estaciones meteorológicas en la subcuenca del río Babahoyo, facilitaría el manejo de la cuenca porque se podría empezar modelar eventos de precipitaciones en donde en función al tiempo de concentración se podrían precisar estudios hidrológicos e hidráulicos como base del diseño de la infraestructura rural y urbana, especialmente en temas de drenajes agrícolas, alcantarillas, vías, puentes, etcétera.

- Porque la población de la subcuenca es importante, deben considerarse las prioridades de uso de territorio especialmente en aspectos de la gestión de riesgo, esto provocará que las zonas de desarrollo urbano y rural, se planifiquen con modelos diseñados para estas zonas y no con modelos extrapolados del resto del país.

- Que el GAD de Los Ríos y/o la Municipalidad de Babahoyo elaboren en conjunto con los habitantes del territorio de la subcuenca ordenanzas que favorezcan la conservación del suelo y de las áreas que son habitad de vida silvestre (fauna y flora), para que de esa manera no se vea comprometida la sostenibilidad de la cuenca con construcciones civiles y disposición de desechos (basura) en sitios inapropiados para estas labores.

- Conservar la protección de la calidad de las aguas; la Prefectura en uso de sus competencias constitucionales para la Gestión Ambiental, de Cuencas y Microcuencas y de Construcción Operación y Mantenimiento de Sistemas de Riego, debe de garantizar la calidad de las aguas de riego y de consumo humano. Sobre todo, debe capacitar a los habitantes sobre todo rurales 
para que reutilicen las aguas negras por ejemplo en riego, claro no sin antes haber hecho el proceso de purificación agroecológica de esas aguas con totoras (Schoenoplectus californicus) con el propósito de disminuir los coliformes presentes, de esa manera se haría un aprovechamiento eficiente del agua en la producción agropecuaria en la subcuenca del río Babahoyo.

- Ejecutar un plan de monitoreo de las fuentes de agua en la subcuenca del río Babahoyo para conocer periódicamente los niveles de contaminación de los afluentes de esta subcuenca, identificando así el o los focos de contaminación y de esa manera buscar y aplicar soluciones efectivas.

- Establecer nuevos sistemas productivos para sustituir la matriz actual de producción agropecuaria la cual es dependiente de agrotóxicos y de extensas superficies de monocultivo.

- Promover el uso de insumos de origen botánico y/o agroecológico en la producción de cultivos, con el propósito de remplazar de manera sistémica los productos agrotóxicos.

- Promover la rotación de cultivo y los cultivos asociados, con la finalidad de este restauraría el equilibrio ecológico en los agroecosistemas del territorio.

- Procurar manejar adecuadamente la actividad ganadera considerando un aspecto fundamental como lo es la capacidad de carga, de manera que no se sigan viendo afectados los sistemas agrosilvopastoriles de la subcuenca.

- Realizar labranza conservacionista en los sistemas de producción de ciclo corto, poner en práctica este tipo de labranza tendría como resultados la conservación del recurso agua y suelos ya que se mitigaría la erosión del suelo en el territorio de la subcuenca del Babahoyo.

- Promover una intensiva educación ambiental en la subcuenca del río Babahoyo, de ese modo, el manejo de los residuos orgánicos e inorgánicos del territorio se realizaría de forma mucho más eficiente.

- Conservar la biodiversidad biológica y genética de la zona promoviendo el uso de variedades nativas de los productos agropecuarios que se cultivan comúnmente, así como el uso y la propagación de especies forestales también nativas de este territorio.

\section{REFERENCIAS}

[1] O. G. Caicedo-Camposano, C. E. Balmaseda-Espinosa, J. E. Tandazo-Garcés, E. M. Layana-Bajaña, and V. L. Sánchez-Vásquez, "Water quality for irrigation of san pablo river, babahoyo municipality, ecuador," Revista Ciencias Técnicas Agropecuarias, vol. 28, no. 3, 2019.

[2] CEPAL, Caracterización de la demanda laboral en el Ecuador con información administrativa. 2018.

[3] E. Patzelt, Fauna del Ecuador. Quito: Grupo Social Fepp, fondo Ecuatoriano Populorum Progressio, 4. ed., corr. y aumentada ed., 2004. OCLC: ocn181767048.

[4] ECURED, "Provincia de Los Ríos (Ecuador) - EcuRed," 2015.

[5] Ministerio del Trabajo, "Rendición de cuentas 2017 «subsecretaria de empleos y salarios».," 2018.

[6] M. Preciado, "Descripción del proyecto de riego y drenaje babahoyo. comunicación personal," 2018.

[7] G. A. D. P. D. L. Ríos, "Plan provincial de riego y drenaje," 2016.

[8] Ministerio de Agricultura, Ganadería, Acuacultura y Pesca, "Parámetros aplicables a la información de planificación institucional: Planes y programas en ejecución - resultados operativos," 2014.

Recibido: 25 de septiembre de 2019

Aceptado: 30 de noviembre de 2019 
